\title{
Newborn cord care practices in Haiti
}

\author{
Susan Walsh ${ }^{\mathrm{a} *}$, Kathleen Norr ${ }^{\mathrm{a}}$, Girija Sankar ${ }^{\mathrm{b}}$, Heather Sipsma ${ }^{\mathrm{a}}$ \\ ${ }^{a}$ Department of Women, Children and Family Health Science, University of Illinois at Chicago, \\ Chicago, Illinois, USA; ${ }^{b}$ Global Health Action, Decatur, Georgia, USA
}

(Received ; accepted

*Corresponding author. Email: $\underline{\text { swalsh@uic.edu }}$

Newborn cord infections commonly lead to neonatal sepsis and death, particularly in low resource countries where newborns may receive unhygienic cord care. Topical application of chlorhexidine to the newborn's cord has been shown to prevent infection. Such benefits may be particularly important in Haiti. We sought to explore current cord care practices by conducting a qualitative study using five focus groups among key community stakeholders (mothers of newborns/children under age two, pregnant women, traditional birth attendants, community health workers, traditional healers) in PetitGoâve, Haiti. Data collection was guided by the Health Belief Model. Results suggest community stakeholders recognize that infants are susceptible to cord infection and that cord infection is a serious threat to newborns. Long-held traditional cord care practices are potential barriers to adopting a new cord care intervention. However, all groups acknowledged that traditional practices could be harmful to the newborn while expressing a willingness to adopt practices that would protect the newborn. Results demonstrate potential acceptability for altering traditional cord care practices among 
neonatal caretakers in Haiti. An informational campaign designed to educate local health workers and new mothers to eliminate unhygienic cord applications while promoting chlorhexidine application may be a strong approach for preventing neonatal cord infections.

Keywords: cord care practices; acceptability; umbilical cord; neonate; Haiti

\section{Introduction}

Globally, approximately seven million children under the age of five die each year (WHO, 2014), and an estimated $44 \%$ of these deaths are concentrated within the first month of life (WHO, 2013). Many of these deaths, however, may be prevented by simple, affordable interventions (Countdown, 2013). One such preventable cause of death is neonatal sepsis, often caused by infections originating in the umbilical cord (omphalitis) (Faridi, Rattan \& Ahmad, 1993; Mir et al., 2011). This infection claims the lives of more than 520,000 newborns around the world every year (Blencowe, Cousens \& Mullany, 2011). Recent research has shown that daily applications of $7.1 \%$ chlorhexidine digluconate, a topical antiseptic delivering $4 \%$ chlorhexidine, to the newborn's umbilical stump during the first week of life can prevent a substantial number of cases of omphalitis and neonatal sepsis in low-income countries (Arifeen et al., 2012; Imdad et al., 2013; Mullany et al., 2006b; Sazawal et al., 2012b; Soofi et al., 2012; WHO, 2009).

Evidence to support the acceptability of chlorhexidine use, however, is limited. Unhygienic and traditional cord care practices are common in many low-resource settings where 
local cultural beliefs and practices are strong influences (Herlihy et al., 2013; Smith, 2009). The recommended use of chlorhexidine requires the discontinuation of many traditional cord care practices as they likely reduce the effectiveness of chlorhexidine to prevent omphalitis (Alam et al., 2008; Mullany et al., 2006a). Recent large scale randomized controlled trials conducted in Bangladesh, Nepal, Pakistan and Tanzania suggest high levels of acceptability of chlorhexidine use among new mothers (Alam et al., 2008; Alper, 2007; Arifeen et al., 2012; Imdad et al., 2013; Mullany et al., 2006 a,b; Mullany et al., 2007; Sazawal et al., 2012b; Soofi et al., 2012). These studies, however, are limited to South Asia and East Africa, and their generalizability to other low-resource settings is unclear.

The benefits of chlorhexidine use may be particularly important in Haiti. Haiti is the poorest country in the Western Hemisphere, and $62 \%$ of the population lives on less than $\$ 1.25$ (US) a day (UNICEF, 2013). The neonatal mortality rate is 25 per 1000 live births (UNICEF, 2013), substantially higher than its island neighbour, the Dominican Republic, and almost three times that of North America (UN, 2011). Although more than 90\% of women in Haiti receive at least one antenatal visit, approximately two-thirds of deliveries occur at home (UNICEF, 2013). Evidence suggests that home births in low-income countries incur a higher risk for cord infection compared with institutional births in developed countries (Imdad et al., 2013). There are, however, no published studies that describe the acceptability of chlorhexidine use for neonatal cord care in Haiti or the potential barriers to adopting a new cord care practice.

Accordingly, we conducted a qualitative study to examine current cord care practices and explore the acceptability of a new cord care practice for the neonate delivered in the community setting in rural Haiti. We used the constructs of the well-established Health Belief Model (HBM) (Rosenstock, Strecher \& Becker, 1988; Janz, Champion, \& Strecher, 2002) to guide the study 
and ensure that we captured multiple aspects of acceptability. The model identifies the following key constructs as critical to fostering the adoption of a new health behaviour: perceived susceptibility, perceived severity, perceived barriers and benefits, and cues to action. The HBM has been used for several decades to increase health-promoting behaviours for a wide variety of health issues in diverse settings.

\section{Methods}

\section{Overview}

We conducted a descriptive qualitative study using five focus groups among key community stakeholders in Petit-Goâve, Haiti within a five-day period in May 2013. A descriptive qualitative study allows the researcher to stay close to the original language and context of the participants in order to capture the meaning of events from their perspective (Sandelowski, 2000; Sandelowski, 2010).

\section{Setting}

Petit-Goâve, Haiti, with a population of 157,296 , is located on the southwestern peninsula, 95 km from the capital, Port-Au-Prince (UN, 2011). Petit-Goâve is divided into twelve communal sections. Global Health Action (GHA), a non-profit organization that has been conducting community-based health and development programs in Haiti since 1980, serves the district (GHA, 2014). GHA has existing health care provider networks throughout the area to serve basic health and development needs of poor rural Haitians. Traditional birth attendants (TBAs) have been trained and supported by the organization. GHA also provides monthly refresher training sessions for the TBAs, who assist in home-based deliveries in Petit-Goâve using clean delivery 
kits. The TBAs work in eight of twelve sections and assisted 848 home-based deliveries in 2013. GHA also trains community health workers (CHWs) who routinely visit mothers and neonates during the first few weeks after delivery. CHWs also provide ongoing health services in the community, including routine immunizations, micronutrient distribution, infant growth monitoring, and health education sessions on sanitation and hygiene promotion and nutrition (GHA, 2014).

\section{Sample}

We conducted five focus groups among key community stakeholders. Mothers of a newborn are the group that would be most directly affected by introducing a new cord care practice such as substituting topical chlorhexidine application for usual cord care and thus we included one focus group consisting of pregnant women. Our second group included pregnant women as well as mothers of children under age two and older women who provide childrearing advice to new mothers (e.g. mother, mother-in-law). We included three other groups: one with TBAs; one with CHWs trained by GHA; one with traditional healers such as the hougan (Vodou priest) or mambo (Vodou priestess) and herbal healers, all who are widely recognized as providing important traditional care in Haiti (Louis, A., 2007). Each focus group was composed of 10-12 participants. All participants were community members and age 18 or older. Pregnant women expecting their first child, mothers of a child under age two and older women who provide childrearing advice to new mothers were recruited from weekly mobile clinics and a local primary health care centre. The community nurse at that clinic screened participants for eligibility, and if eligible, explained to them the opportunity to participate in these focus groups. If women expressed interest, the CHWs and TBAs facilitated their attendance at the designated 
place and time for the assigned focus group. GHA invited CHWs and TBAs to participate in their own focus groups. Traditional healers were invited through the community networking of GHA health workers.

\section{Focus group guide}

The focus group interview guide was structured using the HBM (Rosenstock, Strecher \& Becker, 1988; Janz, Champion, Strecher, 2002). Participants were asked questions in order to explore the theoretical key constructs (Table 1). We first asked about usual/traditional cord care and the use of traditional healers for newborn care, so their discussion was not influenced by questions regarding a new type of cord care. The focus group guide questions queried, 'what do women do in your community', so the same core guide was appropriate for use by both health workers and community women.

\section{Procedure}

An experienced Haitian facilitator who was fluent in Haitian Creole, French, and English led the focus groups. A GHA staff member assisted the facilitator. The facilitator and GHA staff were trained to ensure they understood the purpose of the study, the informed consent procedures, and the questions to be asked. Refreshments were provided, transportation costs were reimbursed, and mothers received baby blankets as compensation for their time. The TBAs, CHWs, and traditional healers did not receive a gift. The protocol was reviewed and approved by the University of Illinois at Chicago Institutional Review Board (\# 2013-0717). Informed consent was obtained from each participant after being given the opportunity to ask questions and/or opt out of group participation. 


\section{Data analysis}

Focus groups were tape-recorded, compared with notes taken during the interviews, transcribed verbatim, and then translated from Haitian Creole into English by GHA staff using a modified consensus translation procedure (Behling \& Law, 2000). Two bilingual GHA staff translated the transcripts from Haitian Creole to French and then to English for data analysis. The Haitian Creole, French, and English transcripts were reviewed and discrepancies were discussed among the translators and the project manager and resolved by consensus.

We began by considering the constructs of the HBM to organize coding, and then developed codes that fit the ideas expressed within each construct: perceived susceptibility, perceived severity, perceived benefits and barriers, and cues to action. Once a set of codes was developed, one of the co-authors (SW) completed coding independently. As part of the abstraction process, we conducted within-case and cross-case analysis following procedures recommended by Ayres, Kavanaugh, and Knafl (2003), noting which stakeholders expressed the different themes and sub-themes.

\section{Results}

Participants discussed the acceptability of modern health care for their community in terms of the perceived susceptibility of the neonate to omphalitis, the perceived severity of infection, perceived barriers and benefits to adopting a new cord care practice, and cues to action for adopting a new cord care practice to avoid infection.

\section{Perceived susceptibility of the neonate to omphalitis}


All five groups of participants recognized the vulnerability of the newborn's umbilical cord to infection. They cited many symptoms of infection, including redness, bleeding, bad odour, green/yellow pus, stomach pain, poor feeding, and umbilical granuloma (small nodule of firm pinkish-red tissue which is not an infection but can bleed easily). A Vodou priest stated, 'Cord pus, red...that happens to people who give birth at home'.

Participants, however, did not always correctly understand the origin of the threat. For instance, one mother said, 'If the mother doesn't take proper precautions, the wind can get into the umbilical cord and make the situation worse'. Another mother said, 'I saw a baby once, after the umbilical cord had fallen off, we saw that the baby's intestines swelled in that area which caused the baby to die. The mother washed clothes for a living, people said that's what caused the problem'. And finally, a TBA described a traditional belief: 'When a matron (TBA) is done cutting the cord of an infant, she should not sleep with her husband, because if she does then the baby will have this fleshy bit (granuloma) on the umbilicus'.

\section{Perceived severity of infection}

Participants from all groups suggested that problems arising from cord infections could be quite severe. One mother stated, 'We take care so that the umbilicus is not wet or moist and does not take air; this could increase the volume of the abdomen and even cause the baby's death'. All groups suggested that they recognized danger signs of illness in the newborn and that they would get the baby to the hospital for serious conditions such as infection, fever, poor eating or sleeping, irritability, distended abdomen, or seizures. A CHW, who typically examines the newborn within the first week of life, confirms that cord infections are one of the conditions for 
which they would seek the highest form of care available: 'Pus and the umbilical cord doesn't dry, you take the baby to the hospital'.

\section{Perceived barriers and benefits to adopting a new cord care practice}

Traditional practices related to cord care may be barriers to adopting new cord care protocols.

Participants in all groups expressed the belief that keeping the neonate's cord covered either by a substance or a cloth of any kind protects against evil spirits carried in the air [wind]. Participants

described several substances traditionally applied to the cord, such as burnt nutmeg, dirt from the threshold of their home, crushed charcoal, ash, burned cotton, palm oil, recipe of leaves and animal excrement, which they identified as healing or protective or both. One woman stated, 'We usually use goat scat; we mix it up with cloves and then apply it on the baby's cord... we do that when the baby's head is open [when the fontanels are not yet closed]'. Another woman suggested, 'If the baby's stomach is swollen, it's common that the mother might wash the abdomen with her urine and give the baby 3 sips of urine to drink - that would work'.

In all groups, many participants suggested the belief that 'wind' (Haitian term) may introduce evil spirits into the neonate's body. A woman emphasized the importance of keeping this 'wind' away from the cord until it separates. 'Powder heals the cord and prevents flies from landing on it. In addition it dries out the cord and keeps winds from getting in which prevents infection'. Vodou priests (hougan or manbo) also spoke of the newborn being 'djoked' (curse, evil spirit) because of open fontanels and/or open umbilical cord which allow the 'djoke' or 'bad air' to enter the body.

Furthermore, cultural and spiritual beliefs greatly influence participants' use of healthcare and may be barriers to the acceptability of new cord care recommendations. Reflecting social 
norms regarding the man as the head and decision-maker of the household, participants in all focus groups referenced the newborn's father as the final decision maker regarding care for the sick neonate. 'I cannot make decisions without my husband'.

Additionally all groups reported either first hand stories or knowing of women in Haiti who rely on Vodou priests or other traditional healers for newborn care. A mother states her belief that the marks on her baby's face came from evil spirits and could only be treated by traditional healers, 'I had a baby once; when I woke up I saw that she had tooth marks (bite marks) on her face; she was covered in blood; in that situation, nobody is going to tell me to take the baby to the hospital'.

Delayed cord separation is also viewed as a potential problem that may interfere with the adoption of new cord care practices; this delay may be particularly relevant to chlorhexidine application to the newborn's umbilical cord stump as it has been shown to lengthen cord separation time for some newborns (Mullany et al., 2006a). One mother states, 'When it's taking too long for the umbilical cord to fall off, I take a little dusting of dirt from in front of the door and I put it on the cord to help dry it out'. Another mother described, 'the baby was [born] at the hospital ...the nurse didn't do anything to the cord, I put powder on the cord when home... took 15 days to fall [off]'.

CHWs shared additional traditional beliefs they have heard from mothers which may interfere with adopting new cord care practices, 'If she sees that a medication isn't working, she could say "I need to put my feet in the water so I don't lose my baby", and then goes to the hougan so she doesn't lose the baby'. Another suggests, 'Sometimes she might throw water into the house, light a candle, make promises thinking that Loa (Vodou spirit) could cause harm'. 
Another CHW said, 'There are parents who go to the hougan with their baby, just to protect it and bathe it, even if the baby isn't ill'.

Although every group described traditional cord care practices that might be barriers to adopting a new cord care practice such as chlorhexidine application, each group also expressed eagerness to obtain modern health care for avoiding and treating neonatal illness, including umbilical cord infection. Traditional healers also discussed the importance of protecting the newborn from evil spirits. When a traditional healer was asked what they would do if an infant was showing danger signs of illness, one traditional healer stated, 'Sometimes, if there is no health centre, the parents will come get us. My first responsibility, if the baby has something I cannot treat, is to refer them to a health centre', Another traditional healer responded, 'It is my first responsibility to get the baby to a health centre'.

Additionally, CHWs and TBAs commented on the perceived benefits of modern medicine versus traditional care for most community members. One CHW explained, 'These days people's eyes are open to [modern] medicine, and when there are problems, people go to the health centre'. Another CHW explained, '... it is true that many eyes are open to the value of [modern] medicine but there are others who aren't'. In response, another CHW stated, 'It is rare these days that a person would see pus... and take her to a hougan'. A TBA reported that if there is a problem with the newborn, 'The mothers will send for us; we will check the baby and if it's beyond us, we will refer to the hospital. Sometimes we accompany them so that they get the attention they need'. Furthermore, both the TBAs and CHWs recognized that traditional cord practices were often unhealthy and should be modified. For instance, one CHW stated, 'If you use dirty gauze it can harm the baby...avoid wetting the umbilical cord because it can cause infection and keep the wound from healing'. Another CHW told about a case where, 'A baby had 
a problem with the umbilical cord. Instead of taking the baby to the health centre...they burned cotton and put it on the baby's cord...it got worse, very grave. She went to the hospital where they cared for the cord and there it healed'.

Cues to action for preventing cord problems. We were unable to explore cues to action specifically for chlorhexidine use since this topical antiseptic was essentially unknown among all groups. One potential cue to action for adopting a new cord care practice, however, is the responsibility TBAs feel for ensuring that their patients are provided with the best care possible. As one TBA states, ' ... if a TBA delivers a baby, they have to check the baby from head to toe... because if something goes wrong, they are going to hold the TBA responsible'. Given this responsibility, the TBAs feel compelled to be responsive to current health recommendations. For example another TBA said that, 'the doctor does not allow us to use goat scat or palm oil'.

A potential second cue to action is the recognition among several of the mothers that traditional cord care practices did not solve cord problems, thus suggesting the need for a more effective alternative. One mother reported a bad experience, 'My cousin went to a hougan and said the baby had a curse on her...took a piece of guaiac wood, lit it on fire and placed it on the baby's cord. I heard "tchoue, tchoue, tchoue" three times... it was those people that caused me to lose my baby. After three days the baby died'. A mother stated, 'We share the information [about our experiences with newborn care] with the women of our community so they are well aware of the problems that they may encounter'. Mothers also reported trusting the health workers in the community, including TBAs, CHWs and the community clinic nurse, to provide the best possible care for their newborns. A mother states, 'First off, the woman has to go to the health centre to get advice on proper nutrition and protection'. These health workers therefore may provide 
important cues to action to mothers, including the recommendation to use chlorhexidine to prevent cord infection and to protect the susceptible newborn.

\section{Discussion}

Our results demonstrate possible acceptability among new mothers in Haiti for adopting a new cord care practice. Participants reported that the infant is susceptible to cord infection, that cord infection was a serious threat to the newborn and that the benefits of modern medicine for avoiding and treating neonatal illness were recognized. However, potential barriers to adopting a new cord care practice were also apparent. Relinquishing traditional cord care practices, such as applying unhygienic substances and covering the cord stump, and a delayed cord separation time may be difficult for women to accept. Despite these potential barriers, all groups also expressed a willingness to adopt practices that would protect the newborn. These results corroborate previous studies that have demonstrated acceptability of a new cord care practice (chlorhexidine application) among new mothers in Pemba, Tanzania (Sazawal et al., 2012a, 2012b); Sylhet, Bangladesh (Alam et al., 2008; Arifeen et al., 20012); southern Nepal (Mullany et al., 2006a; Mullany et al., 2007) and a rural district of Pakistan (Soofi et al., 2012). To our knowledge, our study is the first to explore newborn cord care practices and the potential acceptability of adopting a new cord care practice among women in the Latin American and Caribbean regions.

Our study was conducted in rural communities in one region of Haiti. However, the district of Petit-Goâve is similar to other communities in rural Haiti in demographic composition, household structure, primary occupation, access to and availability of public health services. The practices, understandings and perceptions that are described here may be generalizable only to other rural areas in Haiti with frequent home deliveries. It is not clear how many births occur in 
the community in urban areas of Haiti, as statistics regarding facility versus community deliveries in Haiti do not differentiate between rural or urban settings (UNICEF, 2013).

Our study has several strengths, including the use of native Haitian facilitators for conducting focus groups, involvement of key community stakeholders, and a partnership with Global Health Action (GHA), which has established on-going health promotion initiatives in Haiti. However, it is important to also note its limitations. Our focus groups did not include the views of health professionals, Ministry of Health representatives or professional governing bodies. Although including them would have allowed for a broader system context, this was beyond the scope of our study. Also, these data are representative of one point in time. Although participants in all groups discussed influence of modern medicine on current beliefs and practices, the time frame of these changes cannot be identified from our data.

This study aimed to examine current cord care practices and explore possible acceptability for adopting a new cord care practice such as replacing unhygienic substance application to the newborn's cord with an antiseptic; however, we did not specifically discuss chlorhexidine with all focus group participants. Additional operations research will need to elicit actionable information such as form, dosage, packaging and cost acceptability regarding the use of an antiseptic such as chlorhexidine application. Participants reported their willingness to adopt modern practices that would protect the newborn within a focus group setting, so we cannot rule out the potential for social desirability bias. It must be kept in mind that participants' reporting of acceptability of modern health care may not translate into their actual behaviour of adopting a new cord care practice such as chlorhexidine for newborn cord care.

Recognizing traditional beliefs and practices of newborn cord care is formative prior to introducing a new cord care practice. The local belief system that wind carrying evil spirits can 
enter the newborn's cord and fontanels and cause illness and death is a key concept in attempting to understand the local belief system. Although germ theory appears to be increasingly understood in this community as a result of the cholera epidemic, the belief in 'wind' persists. Because many women did not express opposition to using a new cord care practice but did express a strong belief in using topical applications and coverings to protect the umbilical cord, promoting a new cord care practice such as chlorhexidine application may need to allow the use of hygienic coverings such as a clean porous cloth or gauze pad in conjunction with the antiseptic. Women also believe that a wet cord is undesirable, causing delayed cord separation and leaving the abdomen susceptible [open] to wind. Because chlorhexidine use can prolong cord separation (Mullany et al., 2006a), this could prove to be a barrier in the adoption of chlorhexidine use.

Results of these focus group data suggest that a community-based initiative to establish a new cord care practice such as chlorhexidine application is possible by leveraging an existing model that incorporates active participation and representation from stakeholders at all levels of the health care system. The implementing partner (GHA) has successfully used this model for community-based maternal and child health interventions in this district of Haiti.

A community level campaign focusing on the benefits of adopting a new cord care practice such as chlorhexidine application should begin by informing the community and local health workers including traditional healers. The health workers and healers all expressed a strong desire to protect the newborn from illness. Because of their understanding of traditional practices and beliefs, health workers have opportunities to use their knowledge and influence in the community to promote the use of chlorhexidine in a manner that respects traditional beliefs and practices. In addition, an influence of the newborn's father on health decisions of the neonate 
was identified which could potentially influence different levels of adherence for chlorhexidine daily application.

TBAs, CHWs and clinic nurses may be highly appropriate for promoting a new cord care practice and the application of chlorhexidine. They are respected by community women and routinely visit mothers or see mothers in a community clinic during pregnancy, birth, and the early postpartum period. It would also be important to engage hospital facilities in the area, the Ministry of Health and national health professional associations. Our initiative has begun this networking process. The concept of our community initiative has received the endorsement of The Comite National de Bioethique (a technical group that draws membership from the Ministry of Health and professional medical associations such as the Association Medicale Haitien), the Unite Communale de Santé (a district office of the Ministry of Health), community leadership, private sector facility-based service providers, community-based health providers and implementing organizations. Communications have been initiated with the Haiti Neonatal Alliance. Our research team's participation in the Chlorhexidine Working Group is also indicative of support from the global health community.

Our study has indicated that the introduction of a new cord care practice may be acceptable to both mothers and local caregivers in Haiti because of their strong commitment to newborn health. While long standing traditional cord care practices can be a barrier against adopting a new cord care practice, there is also recognition of potential harm to the neonate from the use of these practices and a potential willingness to accept a new way of caring for the cord that reduces the susceptibility of the neonate to life threatening infections. Local health workers including TBAs, CHWs and traditional healers may be potential resources for facilitating community adoption of a new cord care practice such as the topical application of chlorhexidine. 
The acceptance of non-traditional cord care practices likely has strong implications for improving other aspects of newborn care in Haiti where there is a desperate need of improved neonatal health outcomes. Acceptance among stakeholders for newer healthcare innovations despite their strongly held health beliefs and traditional practices could have implications beyond the prevention of omphalitis and sepsis. Such acceptability for a new cord care practice observed in our study population may also demonstrate that this population might be primed for implementing other new approaches to healthcare. 


\section{Acknowledgements}

This work was supported by Sigma Theta Tau International Honor Society of Nursing: Alpha

Lambda Chapter and Global Health Action. Leslie Ann Cordes, M.D. provided insight and support during the focus group sessions. 


\section{References}

Alam, M.A., Ali, N.A., Sultana, N., Mullany, L.C., Teela, K.C., Khan, N.U.Z., .. Winch, P. J. (2008). Newborn umbilical cord and skin care in Sylhet district, Bangladesh: Implications for the promotion of umbilical cord cleansing with topical chlorhexidine. Journal of Perinatology, 28(Suppl 2), S61-8. doi: 10.1038/jp.2008.164

Alper, B.S. (2007). Antiseptic treatment of umbilical cord reduces omphalitis... and may reduce mortality in developing countries. Clinical Advisor, 10(2), 169-169. Retrieved from http://search.ebscohost.com/login.aspx ?direct=true $\& d b=$ rzh $\& A N=2009520300 \&$ site $=$ $\underline{\text { ehost-live }}$

Arifeen, S.E., Mullany, L.C., Shah, R., Mannan, I., Rahman, S.M., Talukder, M.R., ... Baqui, A.H. (2012). The effect of cord cleansing with chlorhexidine on neonatal mortality in rural Bangladesh: A community-based, cluster-randomized trial. Lancet, 379(9820), 1022-1028.

Ayres, L., Kavanaugh, K., \& Knafl, K.A. (2003). Within-case and across-case approaches to qualitative data analysis. Qualitative Health Research, 13(6), 871-883.

doi:10.1177/1049732303013006008

Behling, O. \& Law, K.S. (2000). Translating questionnaires and other research instruments: Problems and solutions. Thousand Oaks, CA: Sage.

Blencowe, H., Cousens, S., Mullany, L.C., Lee, A.C.C., Kerber, K., Wall, S., .. Lawn, J.E. (2011). Clean birth and postnatal care practices to reduce neonatal sepsis and tetanus: A systematic review and Delphi estimation of mortality effect. BMC Public Health, 11(Suppl 3), S11. doi: 10.1186/1471-2458-11-S3-S11

Faridi, M.M., Rattan, A., Ahmad, S.H. (1993). Omphalitis neonatorum. Journal of the Indian Medical Association, 91(11), 283-285. (Article is not available in electronic format) 
Global Health Action (GHA). (2014). Healthy people, healthy communities, a healthy world.

Retrieved from http://globalhealthaction.org/programs/programs-in-haiti/

Countdown. (2013). Countdown to 2015 maternal, newborn \& child survival: Haiti. Retrieved from http://countdown2015mnch.org/country-profiles/haiti

Herlihy, J.M., Shaikh, A., Mazimba, A., Gagne, N., Grogan, C., et al (2013). Local perceptions, cultural beliefs and practices that shape umbilical cord care: A qualitative study in Southern Province, Zambia. PLOS ONE, 8(11), e79191. doi:10.1371/journal.pone.0079191

Imdad, A., Bautista, R.M.M., Senen, K.A.A., Uy, M.E.V., Mantaring III., J.B., Bhutta, Z.A. (2013). Umbilical cord antiseptics for preventing sepsis and death among newborns. Cochrane Database of Systematic Reviews, 5, CD008635. doi: 10.1002/14651858.CD008635.pub2.

Janz, N.K., Champion, V.L., Strecher, V.J. (2002). The Health Belief Model. In K. Glanz, B. Rimer \& F. Lewis (Eds.), Health behavior and health education: Theory, research and practice (pp. 45-66). San Francisco, CA: Jossey-Bass.

Mullany, L.C., Darmstadt, G.L., Katz, J., Khatry, S.K., LeClerq, S.C., Adhikari, R.K., Tielsch, J.M. (2007). Risk factors for umbilical cord infection among newborns of southern Nepal. American Journal of Epidemiology, 165(2), 203-211. doi: 10.1093/aje/kwj356

Mullany, L.C., Darmstadt, G.L., Khatry, S.K., LeClerq, S.C., Katz, J., Tielsch, J.M. (2006a). Impact of umbilical cord cleansing with $4.0 \%$ chlorhexidine on time to cord separation among newborns in southern Nepal: A cluster-randomized, community-based trial.

Pediatrics, 118(5), 1864-1871. Retrieved from http://search.ebscohost.com/login.aspx?direct=true \&db=rzh\&AN=2009337754\&site= $\underline{\text { ehost-live }}$ 
Mullany, L.C., Darmstadt, G.L., Khatry, S.K., Katz, J., LeClerq, S.C., Shrestha, S., Adhikari, R., Tielsch, J.M. (2006b). Topical applications of chlorhexidine to the umbilical cord for prevention of omphalitis and neonatal mortality in southern Nepal: A community-

based, cluster randomized trial. Lancet, 367(9514), 910-918. doi: 10.1016/S0140$\underline{6736(06) 68381-5}$

Mir, F., Sundar, S., Tikmani, S., Warraich, H., Sultana, S., Ali, S., Zaidi, A. (2011). Incidence and etiology of omphalitis in Pakistan: A community-based cohort study. Journal of Infections in Developing Countries, 5(12):828-833. Retreived from http://web.a.ebscohost.com.proxy.cc.uic.edu/ehost/pdfviewer/pdfviewer?sid=a17822656713-4c65-99eb-6805759ced17\%40sessionmgr4002\&vid=1\&hid=4109

Rosenstock, I.M., Strecher, V.J., \& Becker, M.,H. (1988). Social learning theory and the health belief model. Health Education and Behavior, 15(2), 175-183.

doi:10.1177/109019818801500203

Sandelowski, M. (2000). Focus on research methods-whatever happened to qualitative description? Research in Nursing and Health, 23(4), 334-340. doi: 10.1002/1098240X(200008)23:4<334::AID-NUR9>3.0.CO;2-G

Sandelowski, M. (2010). What's in a name? Qualitative description revisited. Research in Nursing and Health, 33(1), 77-84. doi: 10.1002/nur.20362

Sazawal, S., Dhingra, U., Madhesiya, S., Dutta, A., Ali, S.M., Ame, S., ... Black, R.E. (2012a). Impact of $4 \%$ chlorhexidine cord cleansing of umbilical cord on bacterial growth of newborns in Pemba, Tanzania. Archives of Disease in Childhood, 97, A337. doi:10.1136/archdischild-2012-302724.1177 
Sazawal, S., Dhingra, P., Dhingra, U., Dutta, A., Ali, S.M., Ame, S., .. Black, R.E. (2012b). Trials of improved practices-a pilot study evaluating the acceptability and preferences for a chlorhexidine cord care intervention. Archives of Disease in Childhood, 97, A1. doi:10.1136/archdischild-2012302724.0004

Smith, C. K. (2009). Some traditional umbilical cord care practices in developing countries. Midwifery Today, 91, 12-13. Retreived from http://web.a.ebscohost.com.proxy.cc.uic.edu/chc/pdf?sid=400039bb-d964-45b9-9363f1f619706cfc\%40sessionmgr4005\&vid=1\&hid=4109

Soofi, S., Cousens, S., Imdad, A., Bhutto, N., Ali, N., Bhutta, Z.A. (2012). Topical application of chlorhexidine to neonatal umbilical cords for prevention of omphalitis and neonatal mortality in a rural district of Pakistan: A community-based, cluster-randomized trial. Lancet, 379(9820), 1029-1036. doi:10.1016/S0140-6736(11)61877-1

United Nations (UN). (2011). UN data: A world of information. Retrieved from http://data.un.org/CountryProfile.aspx?crName=Haiti United Nations (UN). (2012). The Millennium Development Goals Report. Retrieved from http://www.un.org/millenniumgoals/pdf/MDG\%20Report\%202012.pdf United Nations Children's Fund (UNICEF). (2013). Statistics at a glance: Haiti. Retrieved from http://www.unicef.org/infobycountry/haiti_statistics.html?p=printme World Health Organization (WHO). (2009). Global health risks. Retrieved from http://www.who.int/healthinfo/global_burden_disease/GlobalHealthRisks_report World Health Organization (WHO). (2013). Children: Reducing mortality (Fact sheet No. 178). Retrieved from http://www.who.int/mediacentre/factsheets/fs178/en/ 
World Health Organization (WHO). (2014). 10 facts on the state of global health. Retrieved from http://www.who.int/features/factfiles/global_burden/en/

Table 1. Focus group guide

Section 1. Potential Barriers (Traditional cord care)

1. Right after the baby is born, how is the cord cut?

2. What do most mothers today do to take care of a newborn baby's cord?

Section 2. Perceived Susceptibility and Severity (Identifying and treating cord problems)

3. Sometimes newborn babies have a problem with the cord stump. What kinds of cord problems have you ever experienced or heard about?

Probes: (for each problem)

a) How common is this?

b) What do mothers do when this happens? [probe for detailed description]

c) What usually happens to the baby?

d) Have you heard of any infant being very sick or dying when there's a problem with the cord?

Section 3. Potential Benefits

4. Have you heard of any things people do to prevent or treat problems with the baby's cord? We want to know about things anybody does, including the mother, her 
relatives, TBAs, and health workers at the clinic and traditional healers (manbo, hougan, Vodou priest/priestess).

\section{Probes:}

You said that people do

a) What is the benefit of doing this? Is it to prevent a problem or treat one?

b) Does the infant's cord [heal better/improve?]

c) How do they do this? [probe for detailed description]

d) Have you heard of any problems for the infant when this is done?

5. Have you heard about using a topical antiseptic to prevent cord infection? If yes, would you be willing to do this?

Section 4. Cues to Action

6. Do mothers take their newborn infant to a community health worker/health care clinic/traditional healer for any medication or treatment?

\section{Probes:}

a) What kinds of infant problems would make a mother take her baby?

b) What does the healthcare worker usually do? 